Cilt: 3

Sayı: 1

Sayfa: $41-48$

Kasım 2016
Volume: 3

Issue: 1

Page: $41-48$

November 2016

\section{VLBI Ölçümlerinden elde edilen VieVS ve Solve UT1 Sonuçlarının Karşılaştırılması}

\author{
Minttu Uunila ${ }^{1, *}$, Karen Baver², John Gipson², Tobias Nilsson³, Hana Krásná4 \\ ${ }^{1}$ Aalto Universitesi, Metsähovi Radyo Gözlemevi, 02540, Kylmälä, Finlandiya \\ ${ }^{2}$ NVI Inc., 7257D Hanover Parkway, 20770, Maryland, ABD \\ ${ }^{3} \mathrm{Helmholtz}$ Centre Potsdam, GFZ Alman Yer Bilimleri Araştırma Merkezi, 14473, Potsdam, Almanya \\ ${ }^{4}$ Viyana Teknik Üniversitesi, Jeodezi ve Jeofizik Enstitüsü, 1040, Viyana, Avusturya
}

Özet

Farklı jeodezik VLBI analiz yazılım paketlerinin karşılaştırılması, bu paketlerin düzgün çalıştıklarından emin olunabilmesi için son derece önemlidir. Viyana VLBI Yazılımı (VieVS) ve Solve, Jeodezik VLBI topluluğu tarafindan kullanılan iki farklı veri yazılım paketidir. Çalışmamızda VieVS ve Solve'dan çaşitli konfigürasyonlarla elde edilen 1 saatlik IVS yoğun ve 24 saatlik R1s ve R4s UT1 ölçüleri incelenmektedir. İki yazılım paketinin ayarları mümkün olduğunca kapsamlı yazılım değişiklikleri yapılmadan senkronize

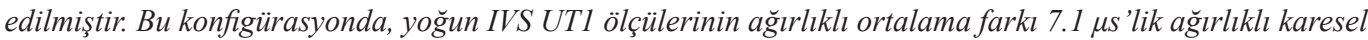
ortalama (WRMS) yayılımı ile 7.8 нs'dir. 24 saatlik ölçülerin ağırlıklı ortalama farkı $5.5 \mu$ s'lik ağırlıklı karesel ortalama (WRMS) yayılımı ile -1.7 $\mu$ s'dir. Ayrıca çözüm konfigürasyonlarının diğer yönlerinin değişmesinin etkileri incelenmiştir. Sonuçları önemli ölçüde kötüleştiren sıkı saat kısıtlamalarının eklenmesi dışında genellikle sonuçların farkları küçüktür.

Anahtar Sözcükler

VLBI, IVS, UT1, Yer dönüklüğü

\section{Abstract \\ Comparison of VieVS and Solve UT1 results from VLBI measurements}

Comparison of different geodetic VLBI analysis software packages is highly important to ensure that they work properly. Two of the data software packages used by the geodetic VLBI community are Solve and the Vienna VLBI Software (VieVS) packages. In our study we investigate UTI estimates from VieVS and Solve for both 1-hour IVS Intensives and 24-hour R1s and R4s with various configurations. We synchronized the settings of the two software packages as much as possible without extensive software changes. In this configuration the weighted mean difference of the UT1 estimates from the IVS intensives was $7.8 \mu$ s with a WRMS scatter of $7.1 \mu \mathrm{s}$. The weighted mean difference for the 24-h sessions was -1.7 $\mu$ s with a WRMS scatter of $5.5 \mu \mathrm{s}$. We also investigated the effect of changing other aspects of the solution configurations. In general the resulting differences were small except for imposing a tight clock constraint which significantly worsened the solutions.

$\underline{\text { Keywords }}$

VLBI, IVS, UT1, Earth rotation

\footnotetext{
** Hakemli değerlendirme sürecinden geçerek, Jeodezi ve Jeoinformasyon Dergisi'nde yayına kabul edilen ve asıl yazı dili İngilizce olan "Comparison of VieVS and Solve UT1 results from VLBI measurements" başlıklı eserin Türkçe diline çevirisidir. Çeviren: Ozan Kılıç (kilic.ozan@metu.edu.tr).

* Sorumlu Yazar:

E-Posta:minttu.uunila@aalto.fi, karen.d.baver@nasa.gov, john.m.gipson@nasa.gov, nilsson@gfz-potsdam.de, hana.krasna@tuwien.ac.at
} 


\section{Giriş}

Bu çalışmada iki yazılım paketinin yer dönüklük parametrelerinin (ERP) ölçümüne yönelik IVS oturumlarından elde edilen ERP tahminleri karşılaştırılmıştır. Uygulamalı bir bakış açısıyla düşünürsek, ERP değerleri GPS uydu navigasyonu gibi uzay aracı navigasyonunda önemli rol oynamaktadır. Bilimsel bir bakış açısıyla ise, ERP değerlerindeki değişiklikler jeofiziksel etkilere bağlıdır ve bu değişimlerin ölçümü çeşitli jeofiziksel modellere kısıtlamalar koymaktadır.

Solve analiz yazılımı (Ma ve diğ. 1990) jeodezik VLBI topluluğu tarafından geniş ölçüde kullanılmaktadır. Daha yeni yazılım paketlerinden biri de Viyana VLBI yazılımıdır (VieVS, Böhm ve diğ. 2009). VieVS Matlab'da yazılmıștır ve genel hatlarıyla OCCAM tabanlıdır. (Titov ve diğ. 2004). VieVS, Solve, C5++ (Hobiger ve diğ. 2010), SteelBreeze (Bolotin 2000) ve OCCAM yazllım paketlerinin hesaplanmış gecikmeleri ve kısmi türevleri Plank ve diğ. (2010) ve Plank (2010) çalışmalarında karşılaştırılmıştır. Araştırmamız özellikle VieVS ve Solve yazılımlarının karşılaştırılmasına odaklandığı, iki yazılım paketinin de konfigürasyonlarını senkronize ettiği, VieVS üzerinde değişiklik yaptığı ve sonuçlarda tek parametre değişikliğinin etkisi üzerinde çalıştığı için Plank ve diğ. tarafından yapılan çalışmaları genişletmektedir.

İki yazılım paketinden elde edilen 1 saatlik yoğun (intensive) IVS ve 24 saatlik IVS-R1 ve IVS R4 oturumlarındaki UT1 ölçüleri karşılaştırılmıştır (Schuh ve Behrend 2012). Daha sonra Uunila ve diğ. (2012) ile daha büyük veri setleri kullanılarak, baz bağımlı ağırlıklar VieVS'e tanıtılarak, yine VieVS'de orta oturum epokları da kullanılarak ve bu çalışmada kullanılan verilerin epoğundan önceki epoklarda meydana gelen büyük depremlerden etkilenen iki VLBI istasyonuna daha çok önem gösterilerek karşılaştırma genişletilmiştir. İstasyonlar: Tsukaba, Japonya'daki 32 metre çapındaki VLBI anteni (TSUKUB32) ve Concepcíon, Şili'deki 6 metre çapında taşınabilir VLBI anteni (TIGOCONC). Tek bir parametrenin değiştirilmesi sonucu oluşan farkları ayrı ayrı gözlemlemek için atmosfer izdüşüm fonksiyonunun değişmesinin, saat kısıtlamasının, öncül EOP dosyasının değişmesinin ve atmosferik basınç yükünün kaldırılmasının etkileri incelenmiştir. Böhm ve Schuh (2007) 1 saatlik oturumlar için UT1 sonuçlarındaki bir izdüşüm fonksiyonunun değiştirilmesinin etkilerini incelemiştir. Fakat bu makalede değinilen 24 saatlik oturumlardaki UT1 sonuçlarını ve diğer tek parametre değişimlerini değerlendirmemiştir.

2. bölüm yazılım paketlerinin yapılandırılmasını, benzerliklerini ve farklılıklarını ve VieVS için yapılan değişikliği açıklamaktadır. 3. bölümde analiz konfigürasyonu, veri setleri ve analiz stratejileri ifade edilmiştir. Bulgular 4. bölümde ve sonuçlar 5. bölümde gösterilmiştir.

\section{Yazılım Paketlerinin Yapılandırılması}

Çalışmamızda VieVS'in 2.2 versiyonu ve Solve'un 21.02.2014 tarihli sürümü kullanılmıştır. İki yazılım paketi de çok esnek yapılandırmalara sahiptir ve sonuçlar seçilen yapılandırma ayarlarına bağlı olarak değişiklik gösterir. $\mathrm{Bu}$ bölümde bu ayarlar tartışılmıştır.

\subsection{Varsayılan Yapılandırma}

\subsubsection{Tanım}

Başlangıç testi olarak yazılımlar Tablo 1'de gösterildiği gibi varsayılan ayarları ile çalıştırılmış, 24 saatlik ve 1 saatlik yoğun UT1 değerleri ayrı ayrı karşılaştırılmıştır. Bu bağlamda varsayılan ayarlarda her iki oturum için yazılımların standart analizlerde kullanılan dosya ve model setleri kullanılmıştır. Modeller, giriş dosyaları ve epoklar VieVS ve Solve'a göre veya 1 saatlik ve 24 saatlik analizlere göre değişiklik gösterebilir. Varsayılan ayarlardan elde edilen çözümlerdeki öncül veriler, aynı öncül EOP verileri kullanılan yazılım paketlerinin ikisinde birden önceden tanımlı olmadığ için tamamıyla varsayılan değildir. Sonuçları yoğun bir şekilde etkilediği için aynı öncül verileri kullanmak önemlidir. Öncül EOP veri değişikliğinin etkisini ölçmek için kullanılan IERS C04 08 hariç, tüm çözümlerimizde USNO'nun finals2000A veri dosyası kullanılmıştır.

Tablo 1 : VieVS ve Solve yazıımlarının varsayılan yapılandırmaları çok benzerdir. Farklııklar belirtilmiştir.

\begin{tabular}{|c|c|c|}
\hline & VieVS ve Solve Varsayılan Yapılandırma & \\
\hline Yazılım & VieVS & Solve \\
\hline $\begin{array}{l}\text { Çözüm biçimi } \\
\text { Oturum sayısı } \\
\text { Efemeris } \\
\text { Basınç Yüklenmesi } \\
\text { Öncül EOP } \\
\text { Presesyon/nutasyon } \\
\text { TRF } \\
\text { CRF } \\
\text { Ağırlıklandırma } \\
\text { İzdüşüm Fonksiyonu } \\
\text { ZWD aralığı, kısıtlama } \\
\text { Saat aralığı, kısıtlama } \\
\text { UT1 aralığı, kısıtlama } \\
\text { Epok }\end{array}$ & $\begin{array}{l}\text { Yalnızca grup gecikmesi } \\
\text { Bir (bağımsız) } \\
\text { JPL } 421 \\
\text { Petrov and Boy (2004) } \\
\text { finals2000A } \\
\text { IAU } 2006 \\
\text { VTRF2008 } \\
\text { Depremler hariç } \\
\text { ICRF2 } \\
33 \text { ps (1cm)'lik sabit değer her bir gözlem hatasına } \\
\text { eklenir } \\
\text { VMF1 } \\
\text { Int: istasyon başına bir ofset, kısıtlama yok } \\
24-h: 30 \text { dakika, } 3.0 \mathrm{~cm} / \mathrm{h} \\
\text { Int: bir offset ve bir oran, kısıtlama yok } \\
24-h: 60 \text { dakika, } 1.3 \mathrm{~cm} / \mathrm{h} \text {, parçalı doğrusal (PWL) } \\
\text { ofsetleri } \\
\text { Int: bir ofset, kısıtlama yok } \\
24-h: \text { bir ofset, kısıtlama yok } \\
\text { gece }\end{array}$ & $\begin{array}{l}\text { Yalnızca grup gecikmesi } \\
\text { Int: Bir (bağımsız) } \\
24-h: \text { birleşik çözüm } \\
\text { JPL } 421 \\
\text { Petrov and Boy (2004) } \\
\text { finals2000A } \\
\text { IAU } 2006 \\
\text { Hesaplanan Öncül TRF } \\
\text { Depremler hariç } \\
\text { Hesaplanan Öncül CRF } \\
\text { Öncül çözümden gelen baz ağırlıkları } \\
\text { VMF1 } \\
\text { Int: istasyon başına bir ofset, kısıtlama yok } \\
\text { 24-h: } 20 \text { dakika, } 0.15 \mathrm{~cm} / \mathrm{h} \\
\text { Int: kısıtlama yok, } 2 \text {. derece bir polinom } \\
\text { 24-h: } 60 \text { dakika, } 0.54 \mathrm{~cm} / \mathrm{h} \text {, ofset, oran, } \\
\text { 2. derece terim } \\
\text { Int: bir ofset, kısıtlama yok } \\
24-h: \text { ofset ve oran, } 3 \mathrm{~ms} / \mathrm{d} \\
\text { orta oturum }\end{array}$ \\
\hline
\end{tabular}




\subsubsection{Karşılaştırma (Benzerlikler ve Farklılıklar)}

Tablo 1'de görüldüğü üzere yazılım paketleri birbirine çok benzemektedir. Bu bölümde bu paketler arasındaki farklılıklar detaylandırılmıştır. Tablo 1 sırasıyla takip edilmiştir.

ERP hesapları öncül istasyon konumlarına çok önemli ölçüde bağlıdır. TIGOCONC (Concepcíon, Şili) ve TSUKUB32 (Tsukuba, Japonya) VLBI istasyonlarının konumları şiddetli depremlerden etkilenmiştir. 27 Şubat 2010'de Şili'de 8.8 (Moment magnitüd ölçeğinde) genişliğinde gerçekleşen deprem sonucu TIGOCONC 3 metre doğu yönünde yer değiştirmiştir (Engelhardt ve diğ. 2011). 11 Mart 2011'de Japonya'da 9.0 (Moment magnitüd ölçeğinde) genişliğinde gerçekleşen Tohoku depremi sonucu TSUKUB32 anteninin UEN yer değiştirmesi $90 \mathrm{~mm}, 640 \mathrm{~mm}$ ve $44 \mathrm{~mm}$ olmuştur. (MacMillan ve diğ. 2012). Bu değişimler sebebiyle bu iki istasyon VieVS ve Solve yazılımlarında özel olarak incelenmiştir.

24 saatlik ve 1 saatlik oturumları işlenirken, Solve öncül TRF olarak, genellikle yakın zamanda deprem görmemiş TRF datum istasyonlarından aldı̆̆ 1 güncel Solve TRF çözümlerini kullanır. Sonrasında TSUKUB32 ve TIGOCONC istasyonlarının konumları, 24 saatlik bir oturum içerisinde ayr1 oturumlara bölünerek hesaplanır. TSUKUB32 için yapılan 1 saatlik yoğun oturumlarda Solve, oturumun epoğundan önceki GPS ölçümlerine dayanan öncül bir konum kullanır. VieVS ise öncül TRF olarak söz konusu epoktan sonra depreme maruz kalmış istasyonlar hariç VTRF2008'i kullanır. Böyle bir durumda VieVS, koordinatlarını istasyon koordinatları için öncül model içeren bir "superstation dosyası"ndan alır. Tutarlılık amacıyla senkronize edilmiş yapılandırmada her iki program da VieVS'in 2012 yılındaki verilerinden hesaplanan parametreleri kullanarak bu istasyonların zamana oranla doğrusal bir şekilde ilerlediğini varsaymaktadir.

VieVS ICRF2'de verilen konumları kullanır. Solve ise genellikle Solve CRF çözümleri arasından en güncel olanı kullanır. ICRF2 ve Solve çözümleri arasında çoğunlukla küçük farklar olmaktadır. Bazı durumlarda sınırlı sayıda yap1lan gözlemler sebebiyle ICRF2 konumları çok iyi bilinemediği için bu fark yüksek çıkabilir. Bu konumlar standart jeodezik oturumların belirlenmesinde kullanılmaz. Bu yüzden en güncel Solve CRF verilerinin kullanılması sonuçları fazla etkilememektedir.

Varsayılan yapılandırma ayarlarında VieVS küresel, Solve ise baz bağımlı ağırlıklandırmaları kullanır. Solve bunun yanında, küresel, baz ve alan bağımlı ağırlıklandırma seçeneklerine de sahiptir. Ağırlıklandırma daha detaylı olarak bölüm 2.2'de tartış1lmıştır.

VLBI analizlerinde troposfer gecikmesinin modellemesi genellikle üç terimle özetlenir: 1.) Hidrostatik gecikme; 2.) Atmosferdeki su buharının sebep olduğu islak gecikme ve 3.) Atmosferik eğikliğin sebebi olan gradyan terimi. Hidrostatik gecikme ve 1slak gecikme, zenit gecikmesinin yüksekliğe bağlı izdüşüm fonksiyonları ile çarpımının birer ürünüdür. Zenit hidrostatik gecikmesi Saastamoinen modeline (Saastamoinen 1972, Saastamoinen 1973) dayalı olarak hesaplanmıştır. Zenit 1slak gecikmesi ise genellikle 20-60 dakika aralıklı parçalı doğrusal fonksiyon (PWL) olarak hesaplanır. Yüksek irtifalarda her iki izdüşüm fonksiyonu da 1/ sin(yükseklik açısı) olarak alınır. Düşük irtifalarda fonksiyonel bağımlılık çok daha karmaşıktır ve genellikle sürekli kesirler halinde verilir. Bu çalışmada doğru izdüşüm fonksiyonunu elde etmek için çalışmalar yapılmış ve değişik izdüşüm fonksiyonlarının etkileri incelenmiştir.

1 saatlik çözümlerde ZWD(Zenit Islak Gecikmesi) iki yazılım paketi için eşdeğer alınmış, 24 saatlik çözümlerde ise ZWD sabitleri değişkenlik göstermektedir.

VieVS 1 saatlik çözümlerinde saatler arasında bir oran (clock rate) ve bir saat ofseti (clock offset) hesaplamaktadir. Solve'un saat hesapları da buna benzemektedir. Solve, 1 saatlik çözümlerinde bir ofset, bir oran ve ikinci derece terim hesaplamaktadır. Bazı 1 saatlik yoğun oturumlarda yaklaşık 18 gözlem yapılmasının planlandığı göz önüne alınırsa, bu ek parametre patolojik olarak kolayca veri kaybına neden olacak az sayıda gözlemle, oturumların hesaplarını biraz zorlaştırarak serbestlik derecesini ortadan kaldırmaktadır.

24 saatlik oturumlarda, her iki yazılım paketi de 60 dakikalık hesaplama aralığı ile PWL saat parametrelendirmesini kullanmaktadır. Fakat kısıtlamalar küçük değişiklikler gösterebilir. VieVS'in bağıl gevşek kısıtlayıcı değeri $1.3 \mathrm{~cm} / \mathrm{h}$ ' dir. Solve'un 1 saatlik oturumlar için kısıtlayıcı değeri ise 0.54 $\mathrm{cm} / \mathrm{h}$ 'ye denk gelen $180 \mathrm{ps} / \mathrm{h}$ değeridir.

1 saatlik çözümlerde iki yazılım paketi de serbest (kısıtlamasız) UT1 ofset hesaplamaları yapmıştır. 24 saatlik çözümlerde Solve $3 \mathrm{~ms} /$ gün kısıtlayıcısı ile bir ofset ve bir oran hesaplarken VieVS varsayılan ayarlarında oturum başına bir ofset hesaplar.

Belirtmek gerekir ki iki yazılım arasındaki tüm farkları araştırmak bu çalışmamızın kapsamı dışında kalmaktadır. Amacımız iki yazılım paketinde de önemli bir değişiklik yapmaksızın yapılandırma ayarlarındaki bazı değişikliklerin UT1 hesaplarına etkilerini incelemektir.

\subsection{VieVS Yazılımındaki Değişiklikler}

VLBI verileri işlenirken $\chi^{2}$ değeri genellikle 4 ve 8 arasında değişiklik göstererek çoğunlukla 1'den büyük olmaktadır. $\mathrm{Bu}$ durum ya çok küçük ölçüm hatalarından ya da verinin yanlış modellenmesinden kaynaklanmaktadır. Bu sorunu ortadan kaldırmak için bir yaklaşım ise $\chi^{2} \sim 1$ yapmak için gözlemdeki hataları arttırarak veriyi yeniden ağırlıklandırmaktır (reweight) (Gipson ve diğ. (2008)).

$\sigma_{t, i, j, o b s}^{2}=\sigma_{t, i j, \text { meas }}^{2}+\varepsilon_{t, i j, w}^{2}$

$\sigma_{t, i j \text {,meas }}^{2} t$ zamanında $i j$ bazındaki ölçüm hatası, $\varepsilon_{t, i j, w}^{2}$ yeniden ağırlıklandırma sabiti, $\sigma_{t, i, j, o b s}^{2}$ ise bunların toplamıdır. Hata eklemek için yaygın olarak 3 yol kullanılmaktadır:

- Küresel yeni ağırlıklar (Global re-weights), tüm gözlemler için aynı;

- İstasyon yeni ağırlıkları (Station re-weights), yalnızca bir gözlemdeki istasyonlara bağl1;

- Baz yeni ağırlıkları (Baseline re-weights), yalnızca bir gözlemdeki bazlara bağl1.

Solve'un işlemsel çözümlerinde varsayılan olarak baz bağımlı ağırlıklar uygulanmıştır. VieVS ise küresel ağırlıklandırma gibi sabit bir ağırlık kullanır ve her bir gözlem hatasına 33 ps $(1 \mathrm{~cm})$ 'lik sabir bir ağırlık ekler. Yazılım paketlerinin yapılandırmalarını birbirine daha fazla benzetmek 


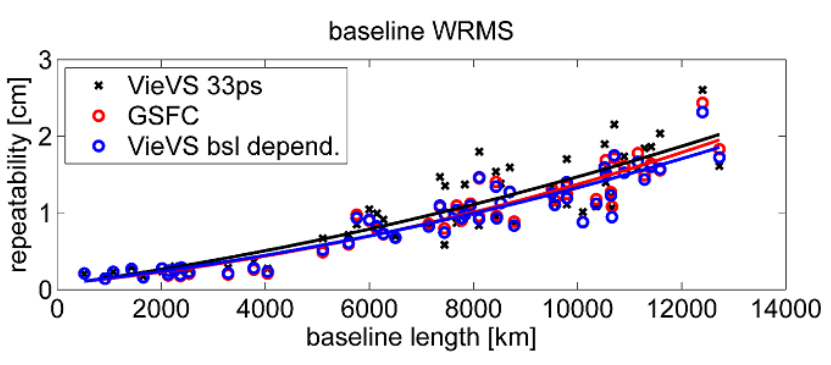

Şekil 1: CONT08 oturumlarındaki VieVS'in orijinal ağırlıklarılla (VieVS 33 ps) elde edilen, Solve'un ağırlık dosyalarıyla (GSFC) elde edilen ve yeni baz bağımlı ağırlıklarla (VieVS bsl depend.) elde edilen baz uzunluk tekrarlanabilirliği.

amaciyla VieVS'de bir gözlemdeki her bir baz için yeni ağırlıklar hesaplandıktan sonra en küçük kareler dengelemesi ikinci bir kez çalıştırılarak tekrarlayan bir işlem oluşturulmuş ve böylece baz bağımlı ağırlıklandırma uygulanmıştır. CONT08 oturumlarındaki analizlerde üç farklı ağırlık planı kullanılarak baz uzunluklarının tekrarlanabilirlikleri hesaplanmıştır (Şekil 1): Solve'un ağırlık dosyaları kullanılarak yapılan orijinal VieVS ağırlıklandırması ve yeni eklenmiş baz bağımlı ağırlıklandırma. CONT08 oturumlarının (Schuh ve Behrend 2012) amacı iki haftalık en gelişmiş VLBI verilerini toplayarak VLBI'ın ne kadar yüksek doğruluklara ulaşabildiğini ispat etmektir. Buna ek olarak yüksek çözünürlüklü yer dönüklük çalışmaları içermektedir. 24 saatlik oturumlardan oluşan 15 günlük CONT08 verilerini gözlemlemek için 11 istasyon Dünya'ya dağıtılmıştır.

Baz bağımlı sabitler ile ağırlıklandırılan gözlemlerle VieVS baz tekrarlanabilirliği, küresel sabitler kullanılarak ağırlıklandırılan gözlemlere oranla önemli ölçüde geliştirilmiştir. Solve'un ağırlık dosyalarıyla yapılan ağırlıklandırmada bazların \%64'ünün ağırlıklı karesel ortalaması (WRMS) gelişmiştir. Baz bağımlı ağırlıklar kullanıldığında ise bazların \%71'inin WRMS değerleri azaltılmıştır. Daha uzun bazlarda etki daha büyüktür. Şekil 2 ve Tablo 4'ten anlaşılacağ üzere baz bağımlı ağırlıklandırmanın kullanılması ayrıca VieVS ve Solve'un 1 saatlik yoğun UT1 hesaplamaları arasındaki uyuşmazlığı da azaltmaktadır. Analizler için 2012 y1lındaki IVS INT1 oturumları (Schuh ve Behrend 2012) kullanılmıştır. INT1 saatlik yoğun oturumları pazartesiden cumaya çalışır ve Kokee Park, ABD ve Wettzell, Almanya arasındaki bazı kullanır.

\section{3 Öncül Yer Dönüklük Parametrelerinin İşlenmesi}

Her iki yazılım paketi de Öncül Yer Dönüklük Parametrelerinin (EOP) ayarında esnek bir yapıya sahiptir. VieVS öncül değerleri bir dosya girdisi olarak alır ve iki seçenek sunar; IERS C04 dosyas1 ya da USNO'nun finals2000A.data dosyası. USNO'nun haftalı güncellenen finals2000A.data dosyası bir yıllık tahminlerle 1 Ocak 1992'den bu yana hesaplanan yer dönüklük verilerini içermektedir (USNO Toshi web sitesi 2014). Ayrıca VieVS, kullanıcıların belirlenen formattaki kendi dosyalarını da kullanmalarına imkan tanır. VieVS öncül epoktan gözlem epoğuna kadar tüm değerleri ya doğrusal interpolasyonu ya da Lagrange interpolasyonunu kullanarak interpole eder. Çalışmamızda varsayılan olarak Lagrange kullanılmıştır.

Solve yazılım paketi de kullanıcılarına belirlenen bir formatta öncül EOP dosyası ekleme olanağı sunar. Eğer herhan- gi bir öncül dosya tanıtılmadıysa, Solve girdinin oturumundaki veri dosyasından aldığı EOP değerlerini kullanacaktır. Fakat bu duruma nadir rastlanır. Petrov ve Baver (2008) 'de de belgelendiği gibi Solve ilk girdiden itibaren gözlem epoğuna kadar tüm verileri interpole etmektedir. İnterpolasyon seçenekleri ucu açık olarak doğrusal, kübik polinom ve kübik spline interpolasyonlarıdır. VieVS ve Solve UT1 bölgesel gelgit değişikliklerini ihmal edebilir ya da dikkate alabilir. Yazılım paketleri bu gelgit değişimlerini "UT1R" (Yoder ve diğ. 1981) ve "UT1S” (Dickman 1993) modellerinin birinden alır. Bu modellerden biri kullanıldığı durumda, Solve interpolasyon başlamadan önce farkları (UT1R-UT1 veya UT1S-UT1) öncül dosyadan çıkarır ve interpolasyon bittikten sonra geri ekler. Solve UT1 ölçümlerinde UT1S modelini ve ucu açık kübik spline interpolasyonunu kullanır. VieVS ise Lagrange interpolasyonunu kullanır. VieVS ayıca bölgesel gelgitlerden kaynaklanan UT1 çeşitliliklerini IERS 2010 toplantısının (Petit ve Luzum 2010) 8. Bölümündeki modeli kullanarak ifade eder. Bu model Yoder ve diğ. (1981) elastik gövde gelgiti, Wahr ve Bergen (1986) sert gövde gelgiti ve Kantha ve diğg. (1998) okyanus gelgit modellerinin bir kombinasyonudur.

$\mathrm{Bu}$ çalışmada VieVS ve Solve tarafından üretilen yer dönüklük değerleri karşılaştırma yapabilmek amacıyla metrik olarak kullanılmıştır ve yazarlar öncül yer dönüklük değerlerini tüm çözümler için tutarlı olarak uygulamıştır. Tüm bu çözümler, C04 08 EOP serilerinde yer değişimini etkisini test etmek amaçlı öncül seri olarak kullanılan tek parametre değişikliği çözümü hariç USNO'nun finals2000A. data dosyasında kullanılmıştır. Solve'un EOP öncül dosyası VieVS'inkine göre bazı değişiklikler içerir; dosyalar farklı formata sahip olmakla birlikte biri diğerine göre önemli bir ek basamak içerir. VieVS değerlerini Solve formatına dönüştürmek için bir yazılım geliştirdik.

\section{Analiz Biçimi}

\subsection{Veri Setleri}

Yer dönüklük parametrelerini (ERP) ölçmek için hazırlanmış iki farklı veri seti kullanarak VieVS ve Solve' dan elde edilen çözümler karşılaştırılmıştır. İlk veri setimiz 2012 yılındaki

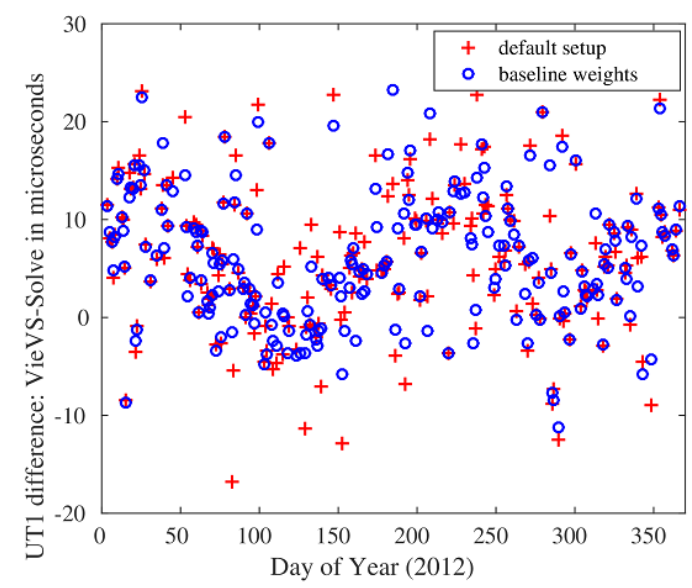

Şekil 2: INT1 1 saatlik oturumlarından elde edilen VieVS - Solve UT1 ölçü farklılıkları. VieVS ilk olarak varsayılan ayarlarında daha sonra baz bağımlı ağırlıklandırma ile orta oturum epoğunda çalıştırılmıştır 
12 veya daha fazla gözlemden oluşan 1 saatlik yoğun gözlemleri barındırır. INT1 saatlik yoğun oturumların altkümesi olarak ayrıca incelenmiștir. INT1'ler Kokee Park, ABD, ve Wettzell, Almanya VLBI istasyonları kullanılarak Pazartesi-Cumartesi aralığında çalıştırılmıştır. Dolayısıyla bunlar kendi içerisinde büyük ve homojen bir veri seti oluştururlar. İkinci veri setimiz ise 2012 yılındaki, büyük UT1 formal hataları bulunan birkaç patolojik durumun dahil edilmediği R1 ve R4 oturumlarını barındırır. "1" ve "4" oturumun sırasıyla Pazartesi veya Perşembe günü başladığını gösterir. R1 ve R4 oturumları için her ağ yaklaşık sekiz istasyona sahiptir. Her gün için artı bir veya iki istasyonu sahip ana bir ăg vardır.

İki veri seti için yazılım paketlerinin UT1 ölçümlerinden elde edilen Ağırlıklı Ortalama (WM) ve Ağırlıklı Karesel Ortalama (WRMS) farkları şu formüller kullanılarak hesaplanmıştır:

$$
W M=\frac{\sum_{i=1}^{N} \frac{\mathrm{UT} 1_{\text {VieVS }, i}-\mathrm{UT1}_{\text {Solve }, \mathrm{i}}}{\sigma_{\text {VieVS }, i}^{2}+\sigma_{\text {Solve }, i}^{2}}}{\sum_{i=1}^{N} \frac{1}{\sigma_{\text {VieVS }, i}^{2}+\sigma_{\text {Solve }, i}^{2}}}
$$

$$
W R M S=\sqrt{\frac{\sum_{i=1}^{N} \frac{\left(\mathrm{UT}_{\text {VieV }, i}-\mathrm{UT1}_{\text {Solve }, i}-W M\right)^{2}}{\sigma_{\text {VieVS }, i}^{2}+\sigma_{\text {Solve }, i}^{2}}}{\sum_{i=1}^{N} \frac{1}{\sigma_{\text {VieV }, i}^{2}+\sigma_{\text {Solve }, i}^{2}}}}
$$

$\mathrm{UT}_{1 \mathrm{VieVS}, \mathrm{i}}$ ve $\mathrm{UT}_{\text {Solve,i }}$ sirasıyla VieVS ve Solve analizlerinden elde edilen UT1 değerleri, $\sigma_{\text {VieVS,i }}^{2}$ ve $\sigma_{\text {Solve,i }}^{2}$ ise bu değerlerin belirsizlikleridir. Sonuçlar Tablo 4'te gösterilmiş ve tartıșılmıştır. Ayrıca her bir UT1 ölçümünün, çeşitlik tekniklerin sonuçlarının toplamından türeyen öncül UT1 serilerinden farkları incelenmiştir. Bu öncüller "gerçek" UT1 değerlerini temsil etmektedir. Bu işlem VieVS ve Solve için ayrı ayrı gerçekleştirilmiştir ve sonuçlar Tablo 5-7 de gösterilmiştir.

\subsection{Analiz Stratejileri}

Çalışmamızda üç set çözüm çalıştırılmıştır: Tablo 2'de gösterildiği üzere Varsayılan, Senkronize Edilmiş ve Tek Değişiklik. Başlangıç olarak her iki yazılım paketi de Tablo 1'de gösterilen Varsayılan çözüm kullanılarak çalıştırılmıştır. Daha sonra üç ana uyuşmazlığı ortadan kaldırmak için çözümler çiftler halinde çalıştırılmıştır. VieVS, Solve'un ağırlık düzenine uymak baz ağırlıklandırmasını kullanmıştır. Orta oturum epokları VieVS'te de kullanılmıştır ve her iki yazılım paketi de TIGOCONC ve TSUKUB32 istasyonlarının koordinatlarını Bölüm 2.1.2'de anlatıldığı gibi kullanmıştır. Çalışmamızda bu çözümler Senkronize Edilmiş olarak adlandırılmıştır. Her bir Senkronize Edilmiş çözümde, bir yön değiştirilirken çözüm yapılandırmasının UT1 ölçümlerine etkisini gözlemlemek amacıyla geri kalan tüm yönler aynı kalmaktadır. Senkronize yapılandırmalara getirilen dört değişiklik: 1) VieVS'de varsayılan izdüşüm fonksiyonunu Küresel İzdüşüm Fonksiyonuna çevirmek, GMF (Böhm ve diğ. 2006b) veya Solve'da (farklı izdüşüm fonksiyonları se-
Tablo 2 : VieVS ve Solve analizlerinde kullanılan üç farklı çözüm yapılandırması

\section{VieVS ve Solve Çözüm Ayarları}

\begin{tabular}{|ll}
\hline Varsayılan & $\begin{array}{l}\text { Her iki yazılım da Tablo 1'de gösterilen varsayılan } \\
\text { ayarları kullanır. }\end{array}$ \\
& $\begin{array}{l}\text { VieVS baz ağırlıklarını ve orta oturum epoklarını } \\
\text { kullanmak üzere değiştirilmiştir. } \\
\text { Solve ve VieVS TIGOCONC ve TSUKUB32 istas- } \\
\text { yon konumlarınu VieVS çözümlerinden alırlar. }\end{array}$ \\
& $\begin{array}{l}\text { Tek değişiklik çözümleri. } \\
\text { Tüm tek değişiklik çözümlere Senkronize Edilmiş } \\
\text { çözümlere göre yapılır. Belirleyici, neyin değiştiğini } \\
\text { belirtir. }\end{array}$ \\
\hline Izdüşüm Fonksiyonu & $\begin{array}{l}\text { VieVS GMF Izdüşüm Fonksiyonunu (Küresel), } \\
\text { Solve NMF Izdüşüm Fonksiyonunu (Niell) kullanır. }\end{array}$ \\
\hline EOP & VieVS ve Solve öncül EOP olarak C04 08'i kullanır. \\
\hline Saat Kısıtlaması & $\begin{array}{l}\text { 24 Saatlik çözümler için her iki yazılım da 0.1 } \\
\text { cm/h'lik kısıtlamayı kullanır. } \\
\text { 1 saatlik yoğun çözümler için her iki yazılım da 1.3 } \\
\text { cm/h'lik kısıtlama kullanır. }\end{array}$ \\
\hline $\begin{array}{l}\text { Atmosfer ve Ok- } \\
\text { yanus Yüklenmesi }\end{array}$ & $\begin{array}{l}\text { Her iki yazılım paketi de atmosferik yükü ve okya- } \\
\text { nus yükünü kullanmaz. }\end{array}$ \\
\hline
\end{tabular}

bebiyle WRMS farkları Tablo 4'ten çıkarılmıştır) Niell İzdüşüm Fonksiyonuna NMF (Niell 1996) çevirmek; 2) öncül EOP dosyasını C04 08'e çevirmek; 3) saat kısıtlama seçeneğini değiştirmek: 24 saatlik oturumlardaki varsayılan VieVS kısıtlamasını 1 saatlik yoğun oturumlar için kullanmak ve 24 saatlik oturum için çok zayıf bir kısıtlama uygulamak; 4) atmosferik basınç yükünü kaldırma. Değişiklikler 1 saatlik ve 24 saatlik her iki oturuma da uygulanmıştır. Değişiklikler ve sonuçlar Tablo 4-7'de listelenmiştir. Karşılaştırma analizlerinde kullanılan varsayılan ve yeni saat kısıtlamaları Tablo 3 ’te gösterilmiştir.

\section{Bulgular}

\subsection{Varsayılan ve Senkronize Edilmiş Çözümler}

Tablo 4 VieVS ve Solve ölçümlerinin farklılıklarını göstermektedir. Varsayılan ayarların sonuçları Tablo 4'ün ilk veri satırında gösterilmiştir. VieVS ve Solve çözümleri arasındaki WRMS değerleri 2012'deki 1 saatlik yoğun ve INT1 oturumları için sırasıyla $8.8 \mu$ s ve $7.4 \mu$ s'dir. Bu, sırasıyla $13 \mu$ s ve $14 \mu$ s civarlarında olan formal hataları (bkz. Tablo 5 ve 6) iyi bir şekilde kıyaslar. 2012'de gözlemlenen tüm 1 saatlik yoğun oturumlar ile INT1 oturumlarının çözümleri arasındaki UT1 farklılıklarının ağırlıklı ortalamaları (WM) sırasıyla $13.7 \mu$ s ve $3.6 \mu$ s'dir. 1 saatlik yoğun veri setleri için WM değerleri daha büyüktür çünkü varsayılan çözümlerde VieVS ve Solve Tsukuba için farklı öncül pozisyonları kullanır. Doğu-Batı bileşenlerindeki farklılıklar UT1 ölçümlerini doğrudan etkileyecektir.

Tablo 4'teki ikinci veri satırı Senkronize Edilmiş çözümlerdeki farklılıkları göstermektedir. VieVS ve Solve arasındaki UT1 farklarının WRMS değerleri 1 saatlik yoğun ve

Tablo 3 : Varsayılan ve tek değişiklik yapılandırmaları için $\mathrm{cm} / \mathrm{h}$ olarak verilen analizlerde kullanılan saat kısıtlamaları

\begin{tabular}{|l|ll|ll|}
\hline \multicolumn{5}{|c|}{ Saat Kısıtlamaları } \\
\hline Çözüm Çeşidi & \multicolumn{2}{|c|}{ 1 Saatlik Yoğun } & \multicolumn{2}{c|}{ 24 Saatlik Oturumlar } \\
\hline Yazılım & VieVS & Solve & VieVS & Solve \\
\hline varsayılan & yok & yok & 1.3 & 0.5 \\
tek değişiklik & 1.3 & 1.3 & 0.1 & 0.1 \\
\hline
\end{tabular}


INT1 oturumları için sırasıyla $7.1 \mu$ s ve $5.2 \mu$ s'dir. Formal hatalar ise sırasiyla $\sim 13 \mu$ s ve $14 \mu$ s'dir. INT1 oturumlarındaki WRMS yayılımları tam yoğunluklu oturumlardakine oranla daha az olduğu tekrar hatırlatılmalıdır. 1 saatlik oturumlar için çözümleri senkronize etmek WM'lerde 13.7 $\mu$ s'den $7.8 \mu$ s'ye kadar büyük bir düşüşe sebep olmuştur. INT1 oturumları için UT1 farklılıklarının WM değerleri aksine $3.6 \mu$ s'den $6.0 \mu$ s'ye yükselmiştir.

24 saatlik oturumlar için, Varsayılan ve Senkronize Edilmiş çözümler arasındaki fark oldukça küçüktür. UT1 farkl1lıklarının WRMS büyüklükleri $5.1 \mu$ s'den $5.5 \mu$ s'ye yükselirken, WM büyüklükleri -2.1 $\mu \mathrm{s}^{\prime}$ den $1.7 \mu$ s'ye düşmektedir. $\mathrm{Bu}$ farkları kayda değer șekilde göz önünde bulundurmuyoruz. 24 saatlik çözümler için formal hata VieVS'de $3.5 \mu \mathrm{s}$, Solve'da $2.8 \mu$ s'dir. Dolayısıyla formal hatalara göre yayılım beklenilenden fazladır. Bu durum çalışmanın daha fazla geliştirilmesi için bir boşluk bırakmaktadır.

Tablo 5,6 ve 7'nin ilk iki satırında C04'e göre 1 saatlik yoğun, INT1 ve 24 saatlik oturumlar için Varsayılan ve Senkronize edilmiş çözümler karşılaştırılmaktadır. Tüm bu veri setleri C04 08 ile karşılaştırıldığında Varsayılan ve Senkronize edilmiş çözümler arasında büyük farklar görülmemektedir.

\subsection{Tek Değişiklik Çözümleri}

Çalıșmamızda ayrıca çözüm ayarlarında tek bir parametrenin değiştirilmesinin etkileri incelenmiştir. Tüm değişiklikler Senkronize Edilmiş çözümlere göre yapılmıştır. Tablo 4 VieVS ve Solve arasındaki farklılıkları göstermektedir. Saat kısıtlaması değiştirilmeden yapılan diğer değișikliklerin WM ve WRMS'e etkisi genel olarak çok düşük, 1 mikro saniye altındadır. Saat kısıtlamasının değiştirilmesinin etkisi çok büyüktür. Örnek olarak, WRMS farkları sırasıyla 1 saatlik yoğun ve INT1 oturumlarda $28.3 \mu$ s'ye $(19.0 \mu \mathrm{s}), 24$ saatlik oturumlarda $6.7 \mu$ s'ye yükselir. İstasyon saatlerindeki hataları doğrudan UT1 ölçümlerime dağıldı ̆g 1 için, saatlerin doğru bir şekilde modellenmesinin önemi vurgulanmıştır.

Tablo 5, tek parametre değişikliğinin VieVS ve Solve'dan elde edilen 1 saatlik yoğun UT1 ölçümler ve C04 08 serileri arasındaki farklara etkisini göstermektedir. Tablo 6 ise INT1 oturumları için aynı bilgileri içermektedir. VieVS çözümlerinde, saat kısıtlama parametresini değiştirmeden farklı bir yapılandırma parametresini değiştirmenin küçük bir etkisi vardır ( $1 \mu$ s altında). Bu RMS yayılımını kayda değer bir şekilde 1 saatlik yoğun oturumlarda $\sim 20 \mu$ s'den 75 $\mu$ s'ye, INT1 veri setinde ise $82 \mu$ s'ye yükseltmektedir. 1.3 $\mathrm{cm} / \mathrm{h}$ değerindeki bir kısıtlama değeri 24 saatlik oturumlarda belirgin bir kötü etki yaratmaksızın normal olarak kullanılmiştır.

Solve çözümleri için, saat kısıtlaması dahil tek bir parametreyi değiştirmek C04 08 ile olan uyumda küçük bir etki oluşturmaktadır. Solve'un saat modeline ikinci derece bir terim eklemenin kısa bir zaman dilimi için sabit bir oran temsil edeceğini düşünmekteyiz. Ayrıca saat kısıtlamasının C04 08'le olan uyumu etkilememesi, dolaylı olarak saat k1sıtlaması eklendiğinde VieVS ve Solve arasındaki WRMS farkının artışının tamamıyla VieVS ölçümündeki bir değişikliğe bağlı olması gerektiğini ifade etmektedir.

INT1 oturumları için, çeşitli VieVS ve Solve çözümleri-
Tablo 4 : Tüm 1 saatlik (INT1'den INT3'e kadar) oturumlar, INT1 oturumları ve 24 saatlik oturumlar için VieVS ve Solve ağırlıklı ortalama (Wm) ve ağırlıkı karasel ortalama (WRMS) UT1 farklııkları. Farklı çözüm ayarları için Tablo 2'yi inceleyiniz.

\begin{tabular}{|c|c|c|c|c|c|c|}
\hline \multicolumn{7}{|c|}{ VieVS-Solve WM ve WRMS farkları $(\mu \mathrm{s})$} \\
\hline \multirow[b]{2}{*}{ Çözüm çeşidi } & \multicolumn{2}{|c|}{$\begin{array}{c}\text { Tüm } 1 \text { saatlik } \\
\text { yoğun }\end{array}$} & \multicolumn{2}{|c|}{ INT1 } & \multicolumn{2}{|c|}{24 saatlik } \\
\hline & WM & WMRS & WM & WMRS & WM & WMRS \\
\hline $\begin{array}{l}\text { Varsayılan ayar } \\
\text { (değişiklik yok) }\end{array}$ & 13.7 & 8.8 & 3.6 & 7.4 & -2.1 & 5.1 \\
\hline $\begin{array}{l}\text { Senkronize } \\
\text { Edilmiş ayar }\end{array}$ & 7.8 & 7.1 & 6.0 & 5.2 & -1.7 & 5.5 \\
\hline EOP: $\mathrm{C} 0408$ & 8.6 & 7.3 & 6.8 & 5.3 & -1.3 & 5.5 \\
\hline $\begin{array}{l}\text { Saat } \\
\text { kısıtlaması }\end{array}$ & 9.4 & 28.3 & 6.1 & 19.0 & -1.4 & 6.7 \\
\hline $\begin{array}{l}\text { Atmosferik } \\
\text { basınç } \\
\text { yüklenmesi } \\
\text { olmadan }\end{array}$ & 7.7 & 7.1 & 6.0 & 5.3 & -1.6 & 5.5 \\
\hline
\end{tabular}

Tablo 5 : 2012'deki tüm 1 saatlik yoğun oturumlar için UT1 ortalama formal hataları ve C04'ten RMS farkları $(\mu s)$

\begin{tabular}{|l|cc|cc|} 
& \multicolumn{3}{|c|}{ VieVS } & \multicolumn{2}{c|}{ Solve } \\
\hline $\begin{array}{l}\text { Varsayılan ayar } \\
\text { (değişiklik yok) }\end{array}$ & 13.1 & 20.3 & 13.2 & 22.5 \\
\hline $\begin{array}{l}\text { UT1 ortalama } \\
\text { formal hata } \\
\text { ayar }\end{array}$ & UT1 RMS & $\begin{array}{c}\text { UT1 ortalama } \\
\text { formal hata }\end{array}$ & UT1 RMS \\
\hline $\begin{array}{l}\text { Izdüşüm fonksiyonu: } \\
\text { GMF/NMF }\end{array}$ & 13.0 & 20.2 & 13.1 & 22.6 \\
\hline EOP: C04 08 & 13.0 & 20.2 & 13.2 & 22.5 \\
\hline Saat kısıtlaması & 33.1 & 74.9 & 13.1 & 21.0 \\
\hline $\begin{array}{l}\text { Atmosferik basınç } \\
\text { yüklenmesi olmadan }\end{array}$ & 13.0 & 20.3 & 13.1 & 21.7 \\
\hline
\end{tabular}

Tablo 6 : 2012'deki INT1 oturumlar için UT1 ortalama formal hataları ve C04'ten RMS farkları ( $\mu$ s)

\begin{tabular}{|l|cc|cc|}
\hline & $\begin{array}{c}|c| \\
\text { UT1 ortalama } \\
\text { formal hata }\end{array}$ & UT1 RMS & $\begin{array}{c}\text { ST1 ortalama } \\
\text { formal hata }\end{array}$ & UT1 RMS \\
\hline $\begin{array}{l}\text { Varsayılan ayar } \\
\text { (değişiklik yok) }\end{array}$ & 14.0 & 20.2 & 14.0 & 20.2 \\
\hline $\begin{array}{l}\text { Senkronize Edilmiş } \\
\text { ayar }\end{array}$ & 14.1 & 20.2 & 14.0 & 20.2 \\
\hline $\begin{array}{l}\text { İzdüşüm fonksiyonu: } \\
\text { GMF/NMF }\end{array}$ & 14.1 & 20.2 & 14.0 & 20.3 \\
\hline $\begin{array}{l}\text { EOP: C04 08 } \\
\text { Saat kısıtlaması }\end{array}$ & 14.1 & 21.0 & 14.0 & 20.4 \\
\hline $\begin{array}{l}\text { Atmosferik basınç } \\
\text { yüklenmesi olmadan }\end{array}$ & 14.1 & 20.3 & 14.0 & 20.8 \\
\hline
\end{tabular}

Tablo 7: 2012'deki IVS R1 ve R4 oturumları için UT1 ortalama formal hataları ve C04'ten RMS farkları ( $\mu \mathrm{s})$

\begin{tabular}{l|cc|cc|} 
& \multicolumn{3}{|c|}{ VieVS } & \multicolumn{2}{c|}{ Solve } \\
\hline $\begin{array}{l}\text { UT1 ortalama } \\
\text { formal hata }\end{array}$ & UT1 RMS & $\begin{array}{c}\text { UT1 ortalama } \\
\text { formal hata }\end{array}$ & UT1 RMS \\
\hline $\begin{array}{l}\text { (değişiklik yok) } \\
\begin{array}{l}\text { Senkronize Edilmiş } \\
\text { ayar }\end{array}\end{array}$ & 3.8 & 6.8 & 2.8 & 5.5 \\
\hline $\begin{array}{l}\text { Izdüşüm fonksiyonu: } \\
\text { GMF/NMF }\end{array}$ & 3.5 & 7.2 & 2.8 & 5.4 \\
\hline $\begin{array}{l}\text { EOP: C04 08 } \\
\text { Saat kısıtlaması }\end{array}$ & 3.6 & 7.6 & 2.8 & 5.6 \\
\hline $\begin{array}{l}\text { Atmosferik basınç } \\
\text { yüklenmesi olmadan }\end{array}$ & 3.6 & 8.4 & 2.8 & 5.4 \\
\hline
\end{tabular}




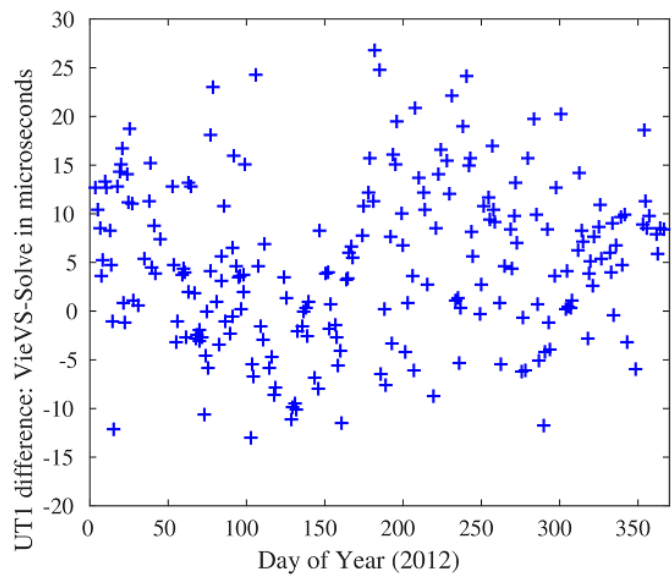

(a) Parametre kestiriminden önce antenlerin koordinatlarına okyanus yüklenme düzeltmeleri getirilmemiştir.

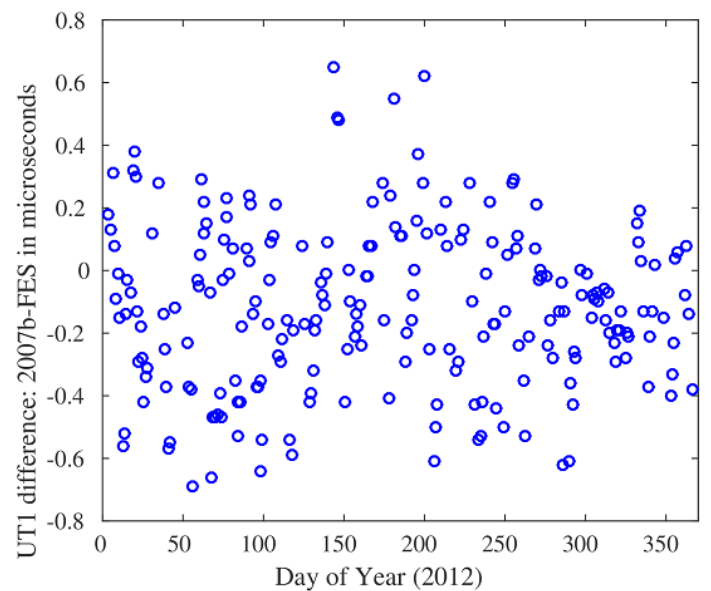

(b) Solve (2007b) - Solve (FES2004)

Şekil 3: (a) INT1 oturumlarındaki çözümlerden elde edilen okyanus yüklenmesi olmadan VieVS - Solve UT1 dengeleme farklılıları (b) Farklı okyanus yüklenme modeli kullanılan iki Solve çözümü arasındaki fark

nin (Saat kısıtlaması çözümleri hariç) C04 08 ile olan uyumları $20 \mu$ s civarında olup neredeyse aynıdır. 1 saatlik yoğun çözümler için Solve WRMS uyumu $22 \mu$ s iken, VieVS WRMS uyumu $20 \mu$ s seviyesindedir. Bu durum VieVS sonuçlarının C04 08'e biraz daha yakın olduğunu göstermektedir.

Tablo 724 saatlik oturumlar için tek bir parametre değişikliğinin etkisini göstermektedir. Genel olarak RMS yay1lımı VieVS çözümlerinde $\sim 7 \mu$ s, Solve'da $5.5 \mu$ s'dir. Bu Solve ölçümlerinin C04 08 ile daha iyi uyum gösterdiğini ifade eder. VieVS çözümlerine odaklanıldığında, saat kısıtlamasının değişimi çoğu parametrenin değişimi yayılımı $\sim 1.2$ $\mu$ s arttırırken, diğer çoğu parametrenin değişimi $\mu$ s altında sonuçlar vermektedir. Solve çözümleri dikkate alındığında ise tek parametre değişikliğinin UT1 ölçümlerine önemli bir etkisi yoktur.

Tablo 4 : Tüm 1 saatlik (INT1'den INT3'e kadar) oturumlar, INT1 oturumları ve 24 saatlik oturumlar için VieVS ve Solve ağırlıklı ortalama (Wm) ve ağırlıklı karasel ortalama (WRMS) UT1 farklılıkları. Farklı çözüm ayarları için Tablo 2'yi inceleyiniz

1 saatlik yoğun çözümler karşılaştırılırken FES2004 okyanus yüklenme modelinin kullanıldığı iki yazılım paketi UT1 dengelemeleri arasındaki farklarda yarıyıllık bir harmonik sinyalle karşılaşılmıştır (Şekil 2 (a)). 1 saatlik oturum çözümleri FES2004 çıkarılıp gelgitsel okyanus yüklenme modeli ile çalıştırıldığında, bu sinyal eskisi kadar belirgin görünmemektedir (Şekil 3 (a)). Şekil 3 (b) iki farklı okyanus yüklenme modelini kullanan Solve çözümleri arasındaki fark1 göstermektedir. 2007b birbirinden farklı modelleri birleştirmektedir (Agnew 1996, Agnew 1997, Petrov ve Ma 2003, Ray 1999). Fark oldukça küçüktür ve belirgin bir harmonik sinyal içermemektedir. Bu, etkinin okyanus yüklenmesinden kaynaklanmadığının bir kanıtıdır. Bu harmonik sinyalin yazılım paketleri arasındaki bir zaman ilişkisinden kaynaklandığını ya da öncüllerin interpolasyonu ile ilişkili olduğunu düşünmekteyiz.

\section{Sonuçlar}

Amaçlarımızdan biri VieVS ve Solve yazılımlarından elde edilen UT1 ölçümlerini karşılaştırmaktı. İki yazılımda da öncül ERP değerleri olarak USNO finals2000A dosyası kullanılarak varsayılan ayarlarda sonuçlar karşılaştırıldı. İkinci olarak ayarlarda olabildiğince eşdeğer değişiklikler yapılan "Senkronize Edilmiş" çözümler çalıştırıldı ve yazılımlara küçük düzeltmeler getirildi. Senkronize edilmiş ayarlarda iki yazılım paketinde de TIGOCONC ve TSUKUB32 istasyonları için aynı öncül TRF koordinatları kullanıldı. Ayrıca VieVS, orta oturum epoklarını ve baz bağımlı ağırlıkları kullanılabilir hale getirildi.

Tablo 4'te belirtildiği üzere VieVS'e baz bağıml ağırlıkları eklemek, iki yazılım paketinde de TSUKUB32 ve TIGOCONC istasyonları için aynı koordinatları kullanmak ve VieVS'in senkronize edilmiş ayarlarında orta oturumları kullanmak önemli sonuçlar vermiştir. Örneğin; tüm 1 saatlik yoğun oturumlardaki UT1 ölçümlerinin WRMS farkları 8.8 $\mu$ s'den $7.1 \mu$ s'ye ve INT1 oturumlardakiler ise $7.4 \mu$ s'den 5.2 $\mu$ s'ye düşmüştür. Senkronize edilmiş ayarlar kullanıldığında 24 saatlik oturumlardaki UT1 ölçümlerinin WRMS farkları $5.1 \mu$ s'den $5.5 \mu$ s'ye yükselerek az miktarda kötüleşmiştir. $\mathrm{Bu}$ WRMS farkları 1 saatlik yoğun oturumlara oranla daha iyi fakat INT1 oturumlarına oranla bir miktar kötüdür. Farklar, VieVS için $4 \mu$ s, Solve için $3 \mu$ s civarında olan formal hatalara oranla biraz daha büyüktür. VieVS'de baz bağım11 ağırlıklar kullanıldığında, baz uzunluk tekrarlanabilirlik WRMS değerleri bazların \%71'inde gelişim göstermiştir.

Çalışmamızda ayrıca çözüm ayarlarını değiştirmenin sonuçlara olan etkisi incelenmiştir. Bunu incelemek için senkronize ayarlarda her aşama için tek bir ayar değiştirilmiştir. Ayarlarda tek bir model veya parametre değiştirildiğinde genellikle VieVS'den elde edilen 24 saatlik çözümlerin sonuçları Solve'a göre daha fazla değişiklik göstermiştir. Saat kısıtlaması seviyesi değiştirilmediği takdirde yapılan değişiklikler sonuçları çok az ( $1 \mu$ s'nin altında) etkilemektedir. Fakat VieVS' de iki oturum çeşidindeki en olumsuz etkiler, saat kısıtlaması 1 saatlik yoğun oturumlar için sıfırdan 1.3 $\mathrm{cm} / \mathrm{h}$ değerine, 24 saatlik oturumlar için $1.3 \mathrm{~cm} / \mathrm{h}$ değerinden $0.1 \mathrm{~cm} / \mathrm{h}$ değerine değiştirildiğinde gözlenmiştir.

Analizler sırasında VieVS ve Solve ölçümleri arasındaki farklarda yarıyıllık harmonik bir sinyale rastlanmıştır. $\mathrm{Bu}$ sinyalin kaynağı hala araştırılmaktadır.

İki yazılım paketinin karşılaştırılması, bu paketlerin düzgün çalıştıklarından emin olunabilmesi için son derece 
önemlidir. Yazılım paketlerindeki küçük değişikliklerin, WRMS farklarına olan etkisinin araştırılması en hassas yer dönüklük parametrelerinin üretilmesi için ayrıca önem taş1maktadır. Gelecekte yükseklik bağımlı ağırlıklandırmanın da (Gipson ve diğ. 2008) VieVS'e tanıtılması yararlı olabilir. 2010 yılındaki IERS toplantılarında tanıtılan ve yazılımların varsayılan ayarlarında kullanılan modellerin kullanılması tavsiye edilmektedir.

VieVS ve Solve farklı yazılımlardır. Fakat farklılıklar çok kolay bir şekilde giderilebilir. Senkronize edilmiş ayarlar tüm 1 saatlik yoğun ve INT1 oturumlarındaki WRMS farklarını önemli bir şekilde azaltmaktadır. Ayrıca, VieVS ve Solve yazılım paketlerinden elde edilen INT1 UT1 RMS ve formal hataları ciddi bir şekilde uyuşmaktadır. Bu durum sonuçların tutarlı olduğunu göstermektedir

\section{Teşekkür}

Bu çalışma NNG12HP00C numaralı NASA sözleşmesi kapsamında desteklenmiştir.

Yazarlar makaleyi geliştirecek olan hakem yorumları için müteşekkirdirler. Makalenin yazarları Goddard Uzay Uçuş Merkezi'nden Dr. D. MacMillan'a, Solve'un kullanımına ilişkin tavsiyeleri ve sağladığı bilgiler için de teşekkür ederler

\section{Kaynaklar}

Agnew D.C., (1997), NLOADF: A program for computing oceantide loading, J. of Geophys. Res, vol. 102, pp. 5109-5110, doi: 10.1029/96JB03458

Agnew D.C., (1996), SPOTL: Some programs for ocean-tide loading, SIO Ref. Ser., 96-9, Scripps Institute of Oceanography, La Jolla, 35p.

Bolotin S., (2000), SteelBreeze home page, http://steelbreeze.sourceforge.net. Accessed on 20 March 2015.

Böhm J., Böhm S., Nilsson T., Pany A., Plank L., Spicakova H., Teke K., Schuh H., (2009), The New Vienna VLBI Software VieVS, In: Proceedings IAG Scientific Assembly 2009, In: International Association of Geodesy Symposia Series Vol. 136, pp 1007-1011.

Böhm J. and Schuh H., (2007), Forecasting Data of the Troposphere Used for IVS Intensive Sessions, In: Proceedings 18th European VLBI for Geodesy and Astrometry Working Meeting, 12-13 April 2007, Vienna, pp 153-157.

Böhm J., Werl B., Schuh H. (2006), Troposphere Mapping Functions for GPS and Very Long Baseline Interferometry from European Centre for Medium-Range Weather Forecasts Operational Analysis Data, Journal of Geophysical Research, 111, B02406, doi: 10.1029/2005JB003629.

Böhm J., Niell A., Tregoning P., Schuh H., (2006), Global Mapping Function (GMF): A New Empirical Mapping Function Based on Numerical Weather Model Data, Geophysical Research Letters 33, L07304, doi: 10.1029/2005GL025546.

Dickman S.R., (1993), Dynamics ocean-tide effects on Earth's rotation, Geophysical Journal International, 11, pp 448-470, doi: 10.1111/j.1365-246X.1993.tb01180.x

Engelhardt G., Thorandt V., Ullrich D., (2011), VLBI Analysis at $B K G$, In: Proceedings 20th European VLBI for Geodesy and Astrometry Working Meeting, 29-31 March 2011, Bonn, pp 102104.

Gipson J., MacMillan D., Petrov L., (2008), Improved estimation in VLBI through better modeling and analysis, In: Proceedings5th International VLBI Service for geodesy and astrometry General Meeting 2008, pp 157-162.

Hobiger T., Otsubo T., Sekido M., Gotoh T., Kubooka T. and Takiguchi H., (2010), Fully Automated VLBI Analysis with c5++ for ultra-rapid determination of UT1, Earth Planets Space, 62, pp 933-937, doi: 10.5047/eps.2010.11.008
Petit G. and Luzum B., (2010), IERS conventions 2010, (IERS Technical Note; 36) Frankfurt am Main: Verlag des Bundesamts für Kartographie und Geodësie, 2010. 179 pp., ISBN 3-89888989-6.

Kantha L.H., Steward J.S. and Desai S.D. (1998) Long-period lunar fortnightly and monthly ocean tides, Journal of Geophysical Research, 103, 12, 639, doi: 10.1029/98jc00888.

Ma C., Sauber J., Clark T., Gordon D., Himwich W.E., Ryan J.W., (1990), Measurement of horizontal motions in Alaska using very long baseline interferometry, Journal of Geophysical Research, 95, B13, 21991-22011, doi: 10.1029/JB095iB13p21991.

MacMillan D., Behrend D., Kurihara S., (2012), Effects of the 2011 Tohoku earthquake on VLBI geodetic measurements, In: Proceedings 7th International VLBI Service for geodesy and astrometry 2012 General Meeting, pp 440-444, NASA/CP-2012-217504.

Niell A.E., (1996), Global mapping functions for the atmosphere delay at radio wavelengths, Journal of Geophysical Research, 101, 32273246, doi: 10.1029/95JB03048.

Petrov L. and Baver K.D., (2008), Description of the keywords of BATCH control language, 7/31/2008

Petrov L. and Boy J.-P., (2004), Study of the atmospheric pressure loading signal in very long baseline interferometry observations, Journal of Geophysical Research, Vol. 109, B3. doi 10.1029/2003JB002500.

Petrov L. and C. Ma, (2003), Study of harmonic site position variations determined by very long baseline interferometry, J. Geophys. Res., 108, 2190, doi: 10.1029/2002JB001801.

Plank L., Böhm J., Schuh H., (2010), Comparison campaign of VLBI data analysis Software - First Results, In: Proceedings 6th International VLBI Service for Geodesy and Astrometry 2010 General Meeting, pp 217-221, NASA/CP-2010-215864.

Plank L., (2010), Results from the VLBI data analysis software comparison campaign, Presented at The First VieVS User Workshop, in Vienna, Austria, 2010.

Ray R.D., (1999), A global ocean tide model from TOPEX/POSEIDON Altimetry: GOT99.2, NASA/TM-1999-209478, Greenbelt, 58 p., 1999.

Saastamoinen J., (1972), Atmospheric correction for troposphere and stratosphere in radio ranging of satellites, The Use of Artificial Satellites for Geodesy, Geophysics Monograph Series, Vol. 15. Edited by Soren W. Henriksen, Armando Mancini, and Bernard H. Chovitz. Washington, DC: American Geophysical Union, 1972., p.247.

Saastamoinen J., (1973), Contributions to the theory of atmospheric refraction, Bulletin Godsique, Volume 47,Issue 1, pp.13-34, doi: $10.1007 / \mathrm{bf} 02522083$.

Schuh H. and Behrend D. (2012), VLBI: A fascinating technique for geodesy and astrometry, Journal of Geodynamics, Vol. 61, pp. 6880. doi: 10.1016/j.jog.2012.07.007.

Titov O., Tesmer V. and B ${ }^{\circ} \mathrm{hm}$ J., (2004), OCCAM v. 6.0 Software for VLBI data analysis, Proceedings 3rd International VLBI Service for Geodesy and Astrometry 2004 General Meeting, pp 267271, NASA/CP-2004-212255.

USNO Toshi web site http://toshi.nofs.navy.mil/ ser7/readme. Accessed on 20 March 2015.

Uunila M., Baver K., Gipson J., Nilsson T., (2012), Comparison of UT1 and polar motion from IVS sessions derived from VieVS and solve analysis, In: Proceedings 7th International VLBI Service for Geodesy and Astrometry General Meeting 2012, pp 400-404, NASA/CP-2012-217504.

Wahr J.M. and Bergen Z., (1986), The effects of mantle anelasticity on nutations, Earth tides, and tidal variations in rotation rate, Geophysical Journal of Royal Astronomical Society, 87(2), pp 633-668, doi:10.1111/j.1365-246X.1986.tb06642.x.

Yoder C.F., Williams J.G. and Parke M.E., (1981), Tidal variations of Earth rotation, Journal of Geophysical Research, 86(B2), pp 881-891, doi:10.1029/JB086iB02p00881. 